Sharif University of Technology
Scientia Iranica
SCIENTIA

\title{
On the orientation dynamics and viscoelasticity of Brownian rigid disklike particles suspended in a shear flow
}

\author{
A. Moosaie* \\ Turbulence Research Laboratory, Department of Mechanical Engineering, Yasouj University, Yasouj, P.O. Box 75914-353, Iran.
}

Received 4 November 2014; received in revised form 15 April 2016; accepted 4 July 2016

\author{
KEYWORDS \\ Dilute suspension; \\ Rigid disklike particle; \\ Direct Monte-Carlo \\ simulation; \\ Moment \\ approximation; \\ Moment closure \\ model; \\ Brownian motion.
}

\begin{abstract}
In this paper, numerical simulations are employed to study the orientation behavior of a dilute suspension of Brownian rigid disklike particles in a simple shear flow. Also, the viscoelasticity of such a suspension is analyzed by considering the stress budget of the two-phase material. A direct Monte-Carlo simulator as well as the moment approximation approaches with two different closure models are used to produce the data. Results are compared by available experimental and analytical data, and a very good agreement is established. After the validation of the simulators, the results are presented and discussed. Different Péclet numbers and shape factors of particles are considered and their effects on various quantities are presented, e.g. particle orientations in space, viscous and elastic contributions to the non-Newtonian stress tensor, etc.
\end{abstract}

(C) 2017 Sharif University of Technology. All rights reserved.

\section{Introduction}

Suspensions of disklike particles are seen in natural and biological systems such as dusts in air and erythrocytes and platelets in blood. These suspensions are also encountered in industrial processes, e.g. manufacturing of cosmetics and ceramics. The dynamic behavior of the suspension depends on the particles orientation distribution. Therefore, it is of paramount importance to accurately predict the particles orientation under the action of different flow conditions. Often, many particles are involved and a statistical description of their orientation distribution is also made possible by the ensemble-averaged moments of the orientation distribution function. These moments can be computed either by solving the complete distribution function or the so-called moment approximation equation. A third approach would be the use of algebraic closure models.

\footnotetext{
*. Tel./Fax: +987433221711

E-mail address: moosaie@yu.ac.ir
}

In the first approach, the evolution of individual fibers orientation is computed using Jeffery's equation [1] to which a stochastic term is added to represent the Brownian motion. Brenner [2] provided analytical solutions for steady shear and extensional flows. Okagawa et al. [3] gave analytical solution to dilute suspension of non-Brownian rigid rodlike and disklike particles subjected to a simple shear flow. However, there exists no closed-form solution to this problem in the general case.

In the numerical context, the stochastic term representing the Brownian motion can be modelled by a Wiener process $[4,5]$. This is equivalent to the Fokker-Planck equation for the distribution function. Then, one can compute the moments of the orientation distribution by an ensemble averaging procedure. Manhart [6,7] adopted such a stochastic method and studied the rheology of fiber suspensions in a turbulent channel flow via one-way coupled simulations. This approach is "exact" in a sense that it does not require any closure model. Moosaie and Manhart [8] proposed 
a direct Monte-Carlo method for the two-way coupled simulation dilute fiber suspension flows and used this approach to study turbulent drag reduction in a channel flow. They used this method to study the structure of vorticity and near-wall partial enstrophy [9] and the pressure-strain correlation [10] in fibrous drag-reduced turbulent channel flow.

Computing the local particle distribution by means of the Fokker-Planck equation requires the numerical solution to a differential equation on the unit sphere for each single particle suspended in the flow simulation domain. As a result, this computation can be prohibitively expensive in real-world timedependent three-dimensional problems, e.g., in turbulent flows [6] or in complex geometries [11]. Thus, Advani and Tucker [12] proposed a second approach: the use of particle orientation tensors, defined as moments of the distribution function. In this approach, known as the moment approximation method, the FokkerPlanck equation is replaced by a hierarchy of moment equations governing the time-evolution of the moments of the orientation distribution function. In each order of the moment approximation, the differential equation for the $2 n$ th-order moment contains $(2 n+2)$ th-order moment. This causes a closure problem. Namely, if one aims at computing the second moment of the orientation distribution function, a closure model expressing the fourth moment in terms of the second moment is required. Among the existing closure models, the linear [13], quadratic, hybrid [14], Hinch and Leal's [15], and the Invariant-Based Optimal Fitting (IBOF) [16] can be mentioned. Chung and Kwon [16] tested their IBOF closure on various problems involving suspension of rigid infinitely long fibers with fiber-fiber interaction. Paschkewitz et al. $[17,18]$ applied the hybrid and IBOF closures to drag reduction in turbulent channel flow using rigid slender fibers. Moosaie et al. [19] proposed a normalization scheme as a closure model to the moment approximation equation. Moosaie et al. [20] derived a closure model based on the reconstruction of the orientation distribution function.

The third approach, called the algebraic closure modeling, is based on algebraic ansatz for the nonNewtonian stress tensor in order to close the NavierStokes equations. Lipscomb et al. [21] made the first attempt in devising a simple algebraic model based on the assumption that fibers align themselves with the local flow velocity vector. Den Toonder et al. [22] adopted this model to study turbulent drag reduction in a pipe caused by polymer additives. This model, called VA (Viscous Anisotropic), revisited by Moosaie and Manhart [23] and extended to VAF (Viscous Anisotropic with velocity Fluctuations), was applied to fulfill more physical constraints and produce more realistic results. Recently, Moosaie et al. [24] have extended the VAF model to take the rotary Brownian motion of particles into account. He used the algebraic model of [23] to directly simulate the fiber-induced turbulent drag reduction in a pressure-driven rodroughened channel flow [25].

Most of the studies, as pointed out above, are devoted to the study of fiber suspensions, and less attention has been paid to suspensions of disklike particles. For example, Yamamoto et al. [26] performed Brownian dynamics simulation of orientation behavior, flow-induced structure, and rheological properties of a suspension of oblate spheroid particles under a simple shear flow.

In the present work, we use the hybrid and IBOF closure models to study the flow-induced orientation distribution in a dilute suspension of Brownian rigid disklike particles suspended in a simple shear flow. We also perform direct Monte-Carlo simulations in the same configuration. As the Monte-Carlo simulator provides the exact solution to the Fokker-Planck equation, its results can be used as reference data. Hence, the objective of this work is to provide some simple benchmark results for further studies and test numerical methods. Moreover, we examine the performance of the hybrid and IBOF closure approximations on the dilute suspension of rigid Brownian disklike particles. The viscoelastic behavior of the suspension is studied as well.

The rest of the paper is organized as follows. In Section 2, the mathematical model of the stochastic and the moment approximation approaches is summarized. The used closure models are explained. Then, the non-Newtonian stress budget is described. The explained theoretical tools are then applied to Brownian disklike particles suspended in simple shear flow in Section 3. The flow-induced orientation distribution, the instantaneous particle distribution, and the non-Newtonian stress budget are studied in detail. The simulations are validated using available analytical solution and experimental data in the literature.

\section{Theory}

In this section, the theory of both stochastic and moment approximation approaches to the dilute suspension of rigid Brownian spheroidal particles is reviewed.

\subsection{Constitutive equations of fiber suspensions}

The stress field caused by an ensemble of rigid particles can be integrated over the stress fields generated by individual particles if the suspension is sufficiently dilute [27]. The hydrodynamic behavior of a single particle has to be known in order to obtain its generated stress field. As the particles are often very small in the context of dilute suspensions, all 
relevant Reynolds numbers are based on the particle size, translational and rotational velocities, and shear rate can be assumed to be small compared with unity. This assumption justifies the neglecting of nonlinear terms in the Navier-Stokes equations; hence, the flow in the vicinity of particles is governed by the quasistatic Stokes equations for creeping flow.

Inertia-free particles, i.e. particles with the same density as the carrier fluid, are convected with the fluid velocity. Brenner [2] showed that only five dimensionless material constants are required to fully describe the hydrodynamic effects of inertia-free particles with axial and fore-aft symmetry. He tabulated these constants for spheroids, long slender axisymmetric bodies and dumbbells, all of which can be derived analytically.

The geometry of disklike particles can be mathematically modelled as an oblate spheroid, as schematically shown in Figure 1. An important geometrical feature of such particles is their aspect ratio, $r=L / D$ (i.e., the ratio of length $L$ to diameter $D$ ). The cases with $r<1$ and $r>1$ represent the oblate and prolate spheroids, respectively, while $r=1$ refers to spherical particles. As the particles are very small, the mechanical forces acting upon them in the flow field of the carrier fluid are not able to cause any substantial deformation. Therefore, an individual disklike particle can be modelled as a rigid body whose orientation is given by unit vector $\mathbf{n}$ perpendicular to the surface of the particle, as shown in Figure 1. The additional stress caused by such a particle in a dilute suspension can be expressed by [6]:

$$
\begin{aligned}
\boldsymbol{\tau}_{\text {part }}= & 2 \mu_{0} \mathbf{D}+\mu_{1} \mathbf{I D}: \mathbf{n n}+\mu_{2} \mathbf{D}: \mathbf{n n n n} \\
& +2 \mu_{3}[\mathbf{n}(\mathbf{D} \cdot \mathbf{n})+(\mathbf{D} \cdot \mathbf{n}) \mathbf{n}]
\end{aligned}
$$

where $\mathbf{D}$ and $\mathbf{I}$ are the strain-rate and the identity tensors, respectively, and $\mu_{0}, \cdots, \mu_{3}$ are material constants. These material constants are functions of

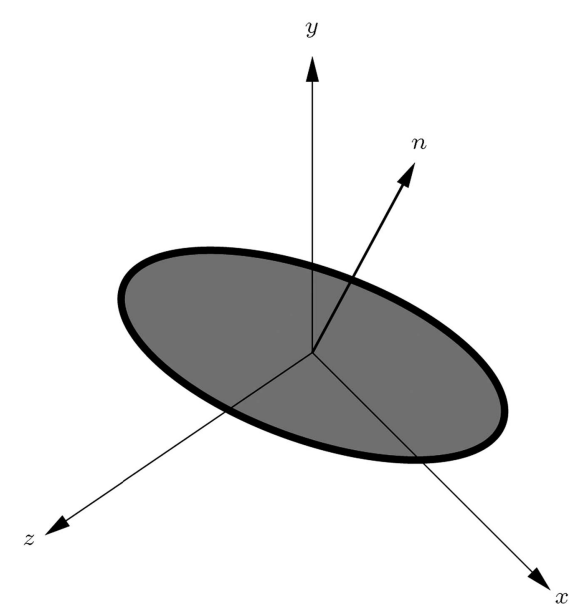

Figure 1. Schematic view of a rigid disklike particle and its orientation vector, $\mathbf{n}$. aspect ratio $r$ and volume fraction $\phi$ of the suspended particles. Strain-rate tensor, D, is defined as the symmetric part of velocity gradient tensor, $\mathbf{L}$, i.e. $\mathbf{D}=$ $\left(\mathbf{L}+\mathbf{L}^{T}\right) / 2$ in which $\mathbf{L}=\nabla \mathbf{u}$ and $\mathbf{u}$ is the fluid velocity field. A rigorous derivation of constitutive Eq. (1) can be found in $[2,15,27,28]$.

In a suspension, each fluid element contains a large number of particles, say $N_{\mathrm{P}}$, with different orientations. The total macroscopic stress is composed of two parts: the Newtonian stress, $\boldsymbol{\tau}^{\mathrm{N}}$, and the nonNewtonian stress, $\tau^{\mathrm{NN}}$, due to the suspended particles.

Newtonian stress, $\tau^{\mathrm{N}}$, is given by the classical relation:

$$
\boldsymbol{\tau}^{\mathrm{N}}=2 \mu \mathbf{D}
$$

where $\mu$ is the molecular viscosity of the carrier fluid. Non-Newtonian stress, $\tau^{\mathrm{NN}}$, is obtained by averaging Eq. (1) over all particles, i.e. $\boldsymbol{\tau}^{\mathrm{NN}}=N_{\mathrm{P}}\left\langle\boldsymbol{\tau}_{\text {part }}\right\rangle$, which for non-Brownian particles gives:

$$
\begin{aligned}
\boldsymbol{\tau}^{\mathrm{NN}}= & 2 \mu_{0} \mathbf{D}+\mu_{1} \mathbf{I D}:\langle\mathbf{n n}\rangle+\mu_{2} \mathbf{D}:\langle\mathbf{n n n n}\rangle \\
& +2 \mu_{3}(\langle\mathbf{n} \mathbf{n}\rangle \cdot \mathbf{D}+\mathbf{D} \cdot\langle\mathbf{n} \mathbf{n}\rangle)
\end{aligned}
$$

Eq. (3) involves the second moment $\langle\mathbf{n n}\rangle$ as well as the fourth moment $\langle\mathbf{n n n n}\rangle$ of the orientation distribution function. Therefore, determination of the nonNewtonian stresses requires a knowledge or estimation of the moments of the orientation distribution of the microstructural conformation. This can be done either by solving the complete distribution function, which is governed by a Fokker-Planck equation or by solving the so-called moment approximation equation.

\subsection{Stochastic approach}

Within the context of a stochastic simulation, the computation of moments of the orientation distribution function always starts with an equation describing the time-evolution of orientation vector, $\mathbf{n}$, for a single isolated particle. Such an equation was derived by Jeffery [1] for rodlike and disklike particles:

$$
\frac{D \mathbf{n}}{D t}=\mathbf{\Omega} \cdot \mathbf{n}+\kappa[\mathbf{D} \cdot \mathbf{n}-(\mathbf{n} \cdot \mathbf{D} \cdot \mathbf{n}) \mathbf{n}]
$$

where $\boldsymbol{\Omega}$ is the rotation-rate tensor, i.e. the asymmetric part of velocity gradient tensor $\boldsymbol{\Omega}=\left(\mathbf{L}-\mathbf{L}^{T}\right) / 2$, and $D / D t=\partial / \partial t+\mathbf{u} \cdot \boldsymbol{\nabla}$ is the material time derivative. Shape factor constant, $\kappa$, solely depends upon the shape of the particle and is defined by:

$$
\kappa=\frac{r^{2}-1}{r^{2}+1} .
$$

Three extreme cases are remarkable; if $\kappa=0$, then the particles have a spherical shape and rotate with the average rotation-rate of the carrier fluid as indicated by 
the term $\boldsymbol{\Omega} \cdot \mathbf{n}$. If $\kappa \rightarrow 1$, then the particles behave like slender rods. The third limiting case is $\kappa \rightarrow-1$, which represents a very thin disklike particle. For $\kappa \neq 0$, the particles' motion depends on the strain-rate of the flow field as well, as indicated by $\kappa[\mathbf{D} \cdot \mathbf{n}-(\mathbf{n} \cdot \mathbf{D} \cdot \mathbf{n}) \mathbf{n}]$.

If the mass of a particle immersed in a fluid is small enough, then it is additionally subjected to Brownian motion due to the thermal fluctuations of the surrounding molecules. Brownian motion of a particle is a random process if considered on the time and length scales of the continuum mechanics, and it can be modelled on the evolution equation of the orientation of a small particle as a random force term $\boldsymbol{\Gamma}(t)$ added to Eq. (4):

$$
\frac{D \mathbf{n}}{D t}=\boldsymbol{\Omega} \cdot \mathbf{n}+\kappa[\mathbf{D} \cdot \mathbf{n}-(\mathbf{n} \cdot \mathbf{D} \cdot \mathbf{n}) \mathbf{n}]+\mathbf{\Gamma}(t) .
$$

Random force term, $\boldsymbol{\Gamma}(t)$, is proportional to Langevin force, $\mathbf{F}_{f}(t)$, divided by mass $m$ of the particle:

$$
\boldsymbol{\Gamma}(t)=\frac{\mathbf{F}_{f}(t)}{m} .
$$

The noise strength of the Langevin force is given by:

$$
\langle\boldsymbol{\Gamma}(t) \boldsymbol{\Gamma}(t)\rangle=q \delta\left(t-t^{\prime}\right) .
$$

That is to say, it is uncorrelated over different times. With Boltzmann constant, $k_{B}$, friction coefficient, $\gamma$, and absolute temperature, $T, q$ is given by:

$$
q=\frac{2 k_{B} T}{\gamma m^{2}} \text {. }
$$

For the rotation of an axisymmetric particle about a transverse axis, the friction coefficient reads:

$$
\gamma=6 \mu V_{P} K_{T}
$$

with $\mu$ being the viscosity of the Newtonian carrier fluid, $V_{P}$ being the particle volume and $K_{T}$ being a material constant [2].

The Langevin force has a formal representation as the increment of a Wiener process:

$$
\mathbf{F}_{f}(t)=\sqrt{2 D_{r}} \frac{\partial \mathbf{W}}{\partial t},
$$

in which the diffusion coefficient is defined by $D_{r}=$ $k_{B} T / \gamma[5]$. The time-dependent orientation of an individual particle subjected to Brownian fluctuations is then given by the following stochastic integral equation:

$$
\mathbf{n}(t)=\mathbf{n}_{0}+\int_{0}^{t} \mathbf{A}\left(t^{\prime}, \mathbf{n}\right) d t^{\prime}+\int_{0}^{t} B d \mathbf{W}_{t}^{\prime},
$$

where:

$$
\begin{aligned}
& \mathbf{A}=\boldsymbol{\Omega} \cdot \mathbf{n}+\kappa[\mathbf{D} \cdot \mathbf{n}-(\mathbf{n} \cdot \mathbf{D} \cdot \mathbf{n}) \mathbf{n}], \\
& B=\frac{\sqrt{2 D_{r}}}{m}
\end{aligned}
$$

This equation is equivalent to the Fokker-Planck equation for distribution function $\Psi(\mathbf{n}, t)$ that describes the fraction of particles in a fluid element with orientation vectors that reside around $\mathbf{n}$ at time $t$ :

$$
\frac{\partial \Psi}{\partial t}=-\boldsymbol{\nabla}_{\mathbf{n}} \cdot\left[\Psi \frac{\partial \mathbf{n}}{\partial t}-D_{r} \boldsymbol{\nabla}_{\mathbf{n}} \Psi\right],
$$

where $\boldsymbol{\nabla}_{\mathbf{n}}$ symbolizes the nabla operator defined on unit sphere $\mathbf{S}$ of $\mathbb{R}^{3}$. This is essentially a conservation equation in orientation space. It was first used by Fokker [29] and Planck [30] to describe the Brownian motion of small particles. It consists of a drift term $-\nabla_{\mathbf{n}} \cdot(\Psi \partial \mathbf{n} / \partial t)$ and the Brownian diffusion term $\boldsymbol{\nabla}_{\mathbf{n}} \cdot\left(D_{r} \boldsymbol{\nabla}_{\mathbf{n}} \Psi\right)$.

In order to compute the non-Newtonian stress tensor due to the particles contributions from Eq. (3), one needs the second and fourth moments of the orientation distribution function. These are weightedaverage quantities over unit sphere, $\mathbf{S}$, defined by:

$$
\langle\mathbf{n} \mathbf{n}\rangle=\oint_{\mathbf{S}} \mathbf{n n} \Psi(\mathbf{n}, t) d S(\mathbf{n}),
$$

$$
\langle\mathbf{n n n n}\rangle=\oint_{\mathbf{S}} \mathbf{n n n n} \Psi(\mathbf{n}, t) d S(\mathbf{n}) .
$$

The Brownian motion not only enters the evolution equation of the particle orientation, but also has to be taken into account in the equation of the nonNewtonian stress tensor generated by the presence of particles:

$$
\begin{aligned}
\boldsymbol{\tau}^{\mathrm{NN}}= & 2 \mu_{0} \mathbf{D}+\mu_{1} \mathbf{I D}:\langle\mathbf{n n}\rangle+\mu_{2} \mathbf{D}:\langle\mathbf{n n n n}\rangle \\
& +2 \mu_{3}(\langle\mathbf{n n}\rangle \cdot \mathbf{D}+\mathbf{D} \cdot\langle\mathbf{n n}\rangle)+2 \mu_{4} D_{r}(3\langle\mathbf{n n}\rangle-\mathbf{I}),
\end{aligned}
$$

where material constant $\mu_{4}$ is given by Brenner [2].

\subsection{Moment approximation approach}

Computing the particles orientation distribution by means of the Fokker-Planck Eq. (15) requires the numerical solution to a differential equation defined on unit sphere, $\mathbf{S}$, for every single particle suspended in the flow simulation domain. This is a prohibitively expensive task for real three-dimensional problems. Therefore, the moment approximation approach is often used instead of a stochastic Monte-Carlo simulation. It offers the opportunity to replace the Fokker-Planck equation with a hierarchy of moment equations. Note that due to the inversion symmetry property of the distribution function, i.e. $\Psi(-\mathbf{n}, \cdots)=$ $\Psi(\mathbf{n}, \cdots)$, all odd-order moments vanish, such that only even-order moments are involved. The hierarchy of evolution equations for the even-order moments is given by Advani and Tucker [12]. Such an equation for the second moment of the orientation distribution 
function is employed in the present work. We use the formulation given by Frattini and Fuller [31] that reads:

$$
\begin{aligned}
\frac{D\langle\mathbf{n} \mathbf{n}\rangle}{D t}= & \mathbf{\Omega} \cdot\langle\mathbf{n} \mathbf{n}\rangle+\langle\mathbf{n} \mathbf{n}\rangle \cdot \mathbf{\Omega}^{T} \\
& +\kappa[\mathbf{D} \cdot\langle\mathbf{n} \mathbf{n}\rangle+\langle\mathbf{n} \mathbf{n}\rangle \cdot \mathbf{D}-2 \mathbf{D}:\langle\mathbf{n n n n}\rangle] \\
& +2 D_{r}[\mathbf{I}-3\langle\mathbf{n} \mathbf{n}\rangle]
\end{aligned}
$$

Eq. (19) takes into account the effect of Brownian motion as reflected by the last term in the right-hand side. At the level of moment approximation equation, it is a damping effect that drags the second moment tensor away from uniaxial state towards isotropic state, $\langle\mathbf{n n}\rangle=\frac{1}{3} \mathbf{I}$, which represents a fully randomized distribution of particles on the unit sphere. The dimensionless number which quantifies the strength of the randomizing effect of the Brownian motion on the particles, compared to the strength of the aligning effect of the external flow, is the local rotary Péclet number defined by:

$$
P e=\frac{\dot{\gamma}}{D_{r}}
$$

where $\dot{\gamma}$ is a characteristic rate of strain of the external flow and is often defined as:

$$
\dot{\gamma}=\sqrt{2 \mathbf{D}: \mathbf{D}}
$$

Eq. (19) contains the fourth moment, 〈nnnn $\rangle$. The evolution equation for any moment will contain terms proportional to the next higher moments [12]. In order to mathematically close the set of equations, a closure model relating $\langle\mathbf{n n n n}\rangle$ to $\langle\mathbf{n n}\rangle$ is thus required. Among all the existing models, the hybrid and IBOF closure approximations are employed in the present work and are reviewed in the next subsection.

\subsection{Closure models}

In the present work, the hybrid and IBOF closure models are used. Their formulations are given below.

1. Hybrid closure (hyb): The hybrid closure approximation of Advani and Tucker [14] reads:

$$
\langle\mathbf{n n n n}\rangle^{\text {hyb }}=\left[(1-f)\langle\mathbf{n n n n}\rangle^{\text {lin }}+f\langle\mathbf{n n n n n}\rangle^{\text {quad }}\right],
$$

where:

$$
\begin{aligned}
\langle\mathbf{n n n n}\rangle^{l \text { in }}= & {\left[-\frac{1}{35}\left(\delta_{i j} \delta_{k l}+\delta_{i k} \delta_{j l}+\delta_{i l} \delta_{j k}\right)\right.} \\
& +\frac{1}{7}\left(a_{i j} \delta_{k l}+a_{i k} \delta_{j l}+a_{i l} \delta_{j k}\right. \\
& \left.\left.+a_{k l} \delta_{i j}+a_{j l} \delta_{i k}+a_{j k} \delta_{i l}\right)\right] \mathbf{e}_{i} \mathbf{e}_{j} \mathbf{e}_{k} \mathbf{e}_{l},
\end{aligned}
$$

$$
\langle\mathbf{n n n n}\rangle^{\text {quad }}=\langle\mathbf{n n}\rangle\langle\mathbf{n} \mathbf{n}\rangle
$$

with $\delta_{i j}$ and $a_{i j}$ being the Kronecker delta and the Cartesian components of $\langle\mathbf{n n}\rangle$, respectively; $\mathbf{e}_{i}$ is the Cartesian basis vectors. Eq. (22) shows that the hybrid closure is a weighted average of the linear and the quadratic closures, and scalar $f$ is to be chosen. In some sense, it will be a measure of particles orientation, since $f=0$ gives an exact closure (linear) if the suspension is isotropic (fully randomized), while $f=1$ gives an exact closure (quadratic) if the particles are fully aligned. Scalar parameter $f$ is often defined as:

$$
f=1-27 \operatorname{det}(\langle\mathbf{n n}\rangle)
$$

in three-dimensional flows.

2. Invariant-Based Optimal Fitting closure (IBOF): The IBOF closure of Chung and Kwon [16] is arguably the best closure available at present. It expresses the symmetric fourth-order tensor $\langle\mathbf{n n n n}\rangle$ as an expansion in the second-order tensor $\langle\mathbf{n n}\rangle$ and identity tensor, $\mathbf{I}$, as derived using the Cayley-Hamilton theorem:

$$
\begin{aligned}
& \langle\mathbf{n n n n}\rangle^{\mathrm{IBOF}}=\beta_{1} \mathbf{S}\{\mathbf{I I}\}+\beta_{2} \mathbf{S}\{\mathbf{I}\langle\mathbf{n n}\rangle\} \\
& +\beta_{3} \mathbf{S}\{\langle\mathbf{n n}\rangle\langle\mathbf{n n}\rangle\}+\beta_{4} \mathbf{S}\{\mathbf{I}(\langle\mathbf{n n}\rangle \cdot\langle\mathbf{n n}\rangle)\} \\
& +\beta_{5} \mathbf{S}\{\langle\mathbf{n n}\rangle(\langle\mathbf{n n}\rangle \cdot\langle\mathbf{n} \mathbf{n}\rangle)\} \\
& \quad+\beta_{6} \mathbf{S}\{(\langle\mathbf{n n}\rangle \cdot\langle\mathbf{n n}\rangle)(\langle\mathbf{n} \mathbf{n}\rangle \cdot\langle\mathbf{n n}\rangle)\}
\end{aligned}
$$

Tensor operator, $\mathbf{S}$, denotes the symmetric part of its argument, $\mathbf{T}=T_{i j k l} \mathbf{e}_{i} \mathbf{e}_{j} \mathbf{e}_{k} \mathbf{e}_{l}$, given by:

$$
\begin{aligned}
\mathbf{S}\{\mathbf{T}\}= & \frac{1}{24}\left[T_{i j k l}+T_{j i k l}+T_{i j l k}+T_{j i l k}+T_{k l i j}\right. \\
& +T_{l k i j}+T_{k l j i}+T_{l k j i}+T_{i k j l}+T_{k i j l} \\
& +T_{j l i k}+T_{l j i k}+T_{j l k i}+T_{l j k i}+T_{i l j k} \\
& +T_{l i j k}+T_{i l k j}+T_{l i k j}+T_{j k i l}+T_{k j i l} \\
& \left.+T_{j k l i}+T_{k j l i}+T_{i k l j}+T_{k i l j}\right] \mathbf{e}_{i} \mathbf{e}_{j} \mathbf{e}_{k} \mathbf{e}_{l}
\end{aligned}
$$

where $T_{i j k l}$ is the Cartesian components of the fourth-order tensor, T. Coefficients $\beta_{1}$ to $\beta_{6}$ are assumed to be functions of the second and third invariants of $\langle\mathbf{n n}\rangle$. These invariants, denoted by $I I$ and $I I I$, are the square and determinant of $\langle\mathbf{n n}\rangle$, respectively. Chung and Kwon [16] derived analytical expressions for three $\beta_{i}$ values and used fifthorder polynomial fittings in terms of the invariants for the remaining three: 


$$
\begin{aligned}
\beta_{1}= & \frac{3}{5}\left[-\frac{1}{7}+\frac{1}{5} \beta_{3}\left(\frac{1}{7}+\frac{4}{7} I I+\frac{8}{3} I I I\right)\right. \\
& -\beta_{4}\left(\frac{1}{5}-\frac{8}{15} I I-\frac{14}{15} I I I\right) \\
& -\beta_{6}\left(\frac{1}{35}-\frac{4}{35} I I-\frac{24}{105} I I I\right. \\
& \left.\left.+\frac{16}{15} I I(I I I)+\frac{8}{35} I I^{2}\right)\right], \\
\beta_{2}= & \frac{6}{7}\left[1-\frac{1}{5} \beta_{3}(1+4 I I)+\frac{7}{5} \beta_{4}\left(\frac{1}{6}-I I\right)\right. \\
& \left.-\beta_{6}\left(-\frac{1}{5}+\frac{4}{5} I I+\frac{2}{3} I I I-\frac{8}{5} I I^{2}\right)\right], \\
\beta_{5}= & -\frac{4}{5} \beta_{3}-\frac{7}{5} \beta_{4}-\frac{6}{5} \beta_{6}\left(1-\frac{4}{3} I I\right),
\end{aligned}
$$

while $\beta_{3,4,6}$ are given by:

$$
\begin{aligned}
\beta_{i}= & a(i, 1)+a(i, 2) I I+a(i, 3) I I^{2}+a(i, 4) I I I \\
& +a(i, 5) I I I^{2}+a(i, 6) I I(I I I) \\
& +a(i, 7) I I^{2} I I I+a(i, 8)(I I) I I I^{2} \\
& +a(i, 9) I I^{3}+a(i, 10) I I I^{3}+a(i, 11) I I^{3} I I I \\
& +a(i, 12) I I^{2} I I I^{2}+a(i, 13)(I I) I I I^{3} \\
& +a(i, 14) I I^{4}+a(i, 15) I I I^{4}+a(i, 16) I I^{4} I I I \\
& +a(i, 17) I I^{3} I I I^{2}+a(i, 18) I I^{2} I I I^{3} \\
& +a(i, 19)(I I) I I I^{4}+a(i, 20) I I^{5} \\
& +a(i, 21) I I I^{5} \\
(i= & 3,4,6) .
\end{aligned}
$$

Twenty one fitting coefficients $a(i, j)$ with $j=$ $1, \cdots, 21$ for each of these three $\beta_{i}$ values were obtained by a least-square which fit with the exact solutions of the Fokker-Planck equation in a variety of simple flows. The values of the fitting coefficients, $a(i, j)$, are provided in an appendix to [16]. They tested their closure model on the suspensions of infinitely long fibers. One of the objectives of the present work is to test the IBOF closure on the suspensions of rigid Brownian disklike particles.

\subsection{Viscoelastic stresses}

Non-Newtonian stress tensor, $\boldsymbol{\tau}^{\mathrm{NN}}$, consists of two parts: a viscous and an elastic contribution [7]:

$$
\tau^{\mathrm{NN}}=\tau^{\mathrm{V}}+\tau^{\mathrm{E}}
$$

where the viscous stress depends upon the current rateof-strain tensor and is given by:

$$
\begin{aligned}
\boldsymbol{\tau}^{\mathrm{V}}= & 2 \mu_{0} \mathbf{D}+\mu_{1} \mathbf{I D}:\langle\mathbf{n n}\rangle+\mu_{2} \mathbf{D}:\langle\mathbf{n n n n}\rangle \\
& +2 \mu_{3}(\langle\mathbf{n n}\rangle \cdot \mathbf{D}+\mathbf{D} \cdot\langle\mathbf{n n}\rangle)
\end{aligned}
$$

and the elastic stress reads:

$$
\boldsymbol{\tau}^{\mathrm{E}}=2 \mu_{4} D_{r}(3\langle\mathbf{n n}\rangle-\mathbf{I})
$$

Phenomenologically, the viscous stress is the stress which instantaneously vanishes when the rate of strain is zero. The elastic stress does not vanish until the system is in equilibrium. It describes the stress due to the Brownian motion. It is the only term that results in stresses which do not linearly depend on the deformation-rate tensor of the surrounding fluid, i.e. it results in stresses in a fluid at rest. It is a consequence of the relaxation of the distribution function towards its isotropic equilibrium due to the Brownian motion. This state of equilibrium is described by the diagonal elements of $\langle\mathbf{n n}\rangle$ taking value $0 . \overline{3}$ and off-diagonal elements vanishing. One can easily verify that the Brownian stress term will disappear as soon as this equilibrium state is reached. The consequences of the Brownian stresses are severe. They change the material properties from a purely viscous behavior to viscoelastic behavior if the Brownian motion is strong enough, i.e. the particles are small enough to be subjected to the Brownian motion. The ensemble of particles will then be able to store energy and release it again at a later instant of time. This energy will be released into the surrounding fluid via the relaxation process described by the elastic stress.

The energy exchange between the carrier fluid and the suspended particles is described by stress power, $S_{p}$, which, according to Eq. (32), consists of a viscous and an elastic part:

$$
S_{p}=\tau^{\mathrm{NN}}: \mathbf{D}=\Phi+\frac{D A^{P}}{D t}
$$

The viscous term is related to additional hydrodynamic energy dissipation, $\Phi$, due to the presence of the particles:

$$
\Phi=\tau^{\mathrm{V}}: \mathbf{D}
$$

The elastic term is related to the change in free energy, $A^{P}$, of the particle system due to the exchange with the carrier fluid:

$$
\frac{D A^{P}}{D t}=\boldsymbol{\tau}^{\mathrm{E}}: \mathbf{D}
$$


While the energy dissipation is always positive, the rate of change of energy can be positive or negative, which accounts for accumulation or release of free energy, respectively.

\section{Disklike particles in a simple shear flow}

In this section, we apply the methods explained in the previous sections to some simple flow cases. Jeffery's equation (Eq. (6)) is advanced in time by an explicit Euler method, while an explicit second-order RungeKutta scheme is employed for the time-advancement of moment approximation (Eq. (19)). The results are compared with the analytical solution of Okagawa et al. [3] for non-Brownian particles and the experiments of Frattini and Fuller [31] for Brownian particles. Once our direct Monte-Carlo simulator is validated, we use its results as reference data in flow cases for which we do not have analytical and/or experimental data.

The measure of error between the exact values obtained from either the analytical solution or the Monte-Carlo simulation and those predicted by the closure approximations is defined as $[32,16]$ :

Tensor components error:

$$
\mathbf{e}=\langle\mathbf{n} \mathbf{n}\rangle^{\text {exact }}-\langle\mathbf{n} \mathbf{n}\rangle^{\text {closure }} \text {. }
$$

Scalar error measure:

$$
e=\sqrt{\frac{1}{2} \mathbf{e}: \mathbf{e}}
$$

The velocity field of the steady planar Couette flow reads $u=\dot{\gamma} z$ as schematically shown in Figure 2. We consider both non-Brownian and Brownian particles here. For non-Brownian particles $(P e \rightarrow \infty)$, the solution is periodic in time. Jeffery [1] gives an analytical expression for the time period:

$$
T=\frac{2 \pi\left(r+r^{-1}\right)}{\dot{\gamma}} .
$$

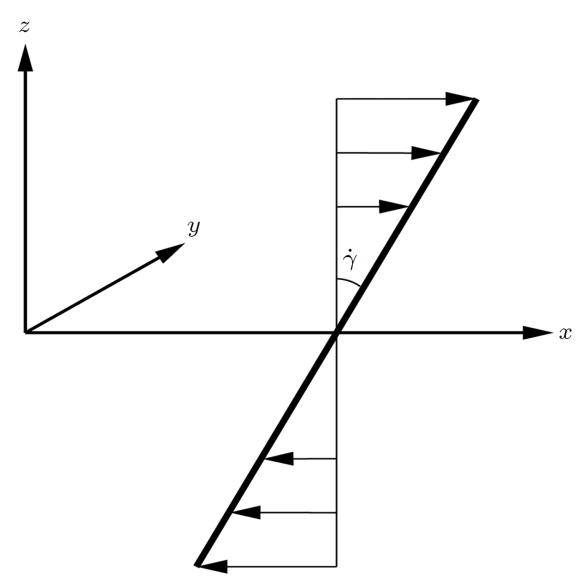

Figure 2. Schematic view of a simple planar shear flow in $x z$-plane.
Okagawa et al. [3] presented an analytical solution for the orientation distribution function of rigid nonBrownian rodlike and disklike particles in a simple shear flow. Their analytical results are used here as reference data to validate our simulations. For $r=0.2$, dimensionless half period, $\dot{\gamma} T / 2$, computed by Eq. (40) is 16.336 . The time period computed by the MonteCarlo simulation and the IBOF closure exactly matches this period. The hybrid model wrongly predicts the time period.

The effect of time step size $d t$ on both direct Monte-Carlo and the moment approximation simulations with IBOF and hybrid closure models is investigated. Figure 3 shows the results of the Monte-Carlo simulation for the evolution of the second moment $\left\langle n_{1} n_{1}\right\rangle$ of the orientation distribution function with different values of $d t$. Figures 4 and 5 show components $\left\langle n_{3} n_{3}\right\rangle$ and $\left\langle n_{1} n_{3}\right\rangle$ of the second moment computed by using the IBOF and hybrid closures, respectively. The results of the Monte-Carlo simulation and the IBOF closure are converging towards the analytical solution

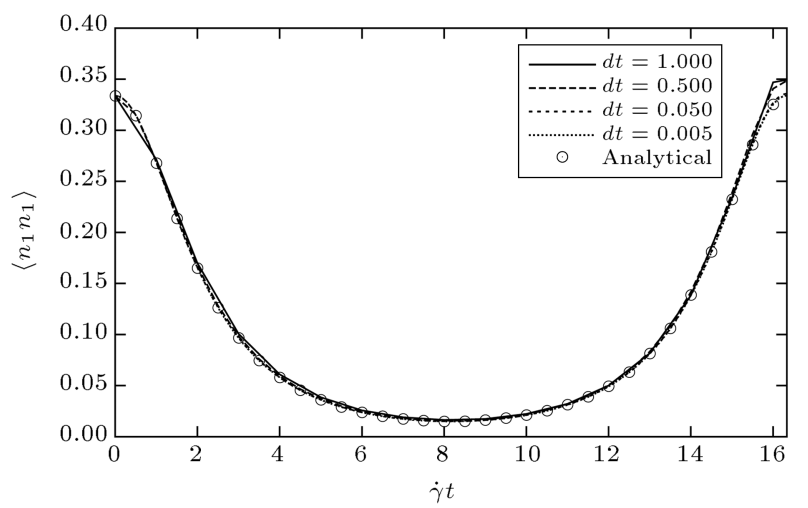

Figure 3. Effect of time step size on the second moment $\left\langle n_{1} n_{1}\right\rangle$ of orientation distribution function of non-Brownian particles with $r=0.2$ by using direct Monte-Carlo simulation.

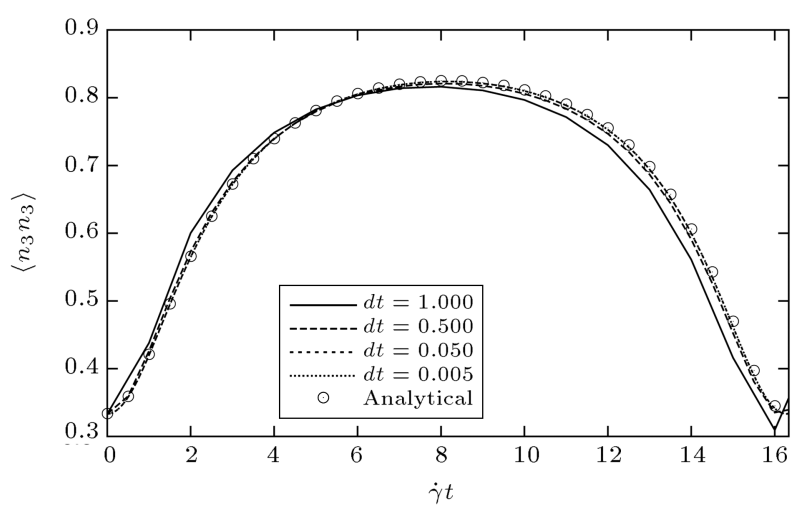

Figure 4. Effect of time step size on the second moment $\left\langle n_{3} n_{3}\right\rangle$ of orientation distribution function of non-Brownian particles with $r=0.2$ by using IBOF closure. 


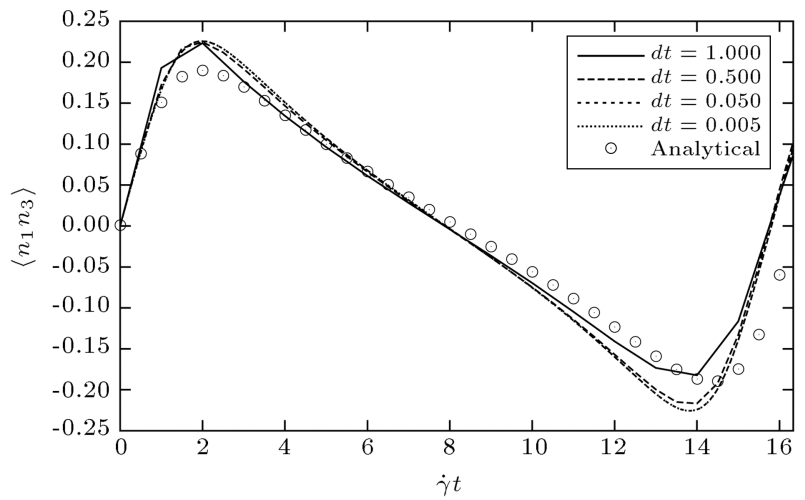

Figure 5. Effect of time step size on the second moment $\left\langle n_{1} n_{3}\right\rangle$ of orientation distribution function of non-Brownian particles with $r=0.2$ by using hybrid closure.

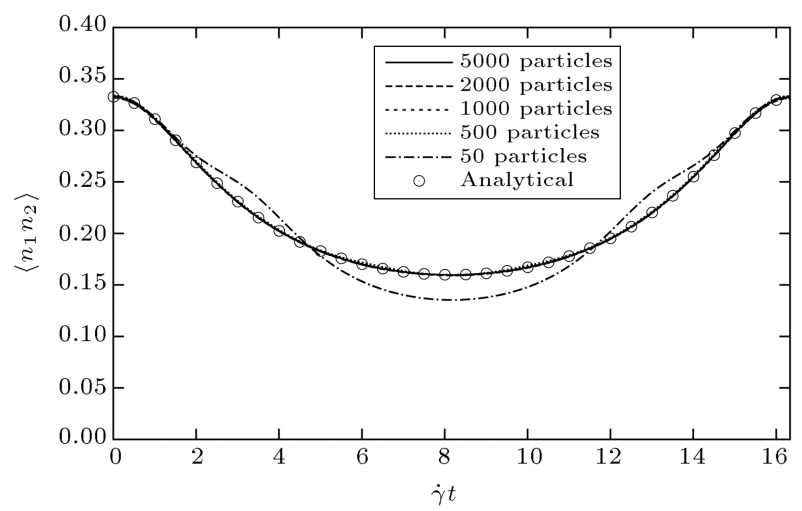

Figure 6. Effect of number of particles on the second moment $\left\langle n_{2} n_{2}\right\rangle$ of orientation distribution function of non-Brownian particles with $r=0.2$ in the Monte-Carlo simulation.

by refining the time step size. The hybrid closure can reproduce neither the amplitude nor the time period accurately.

Figures 3, 4, and 5 demonstrate that the change of the solution between $d t=0.05$ and $d t=0.005$ is very small, and thus, we use $d t=0.005$ hereafter.

In the context of a stochastic simulation, the number of particles is important; an insufficient number of particles lead to non-converged statistics, while redundancy in the number of particles leads to overhead computations. As a result, the influence of the number of particles in the direct Monte-Carlo simulation is investigated. Figure 6 shows $\left\langle n_{2} n_{2}\right\rangle$ component of the second moment of the distribution function resulted from the Monte-Carlo simulation with different numbers of particles. Using 50 particles leads to a considerable deviation from the analytical result. Increasing the number of particles results in a convergence towards the analytical solution. It is shown that with 5000 particles, we obtain converged statistics. Thus, 5000 particles are hereafter used in the Monte-Carlo simulations.

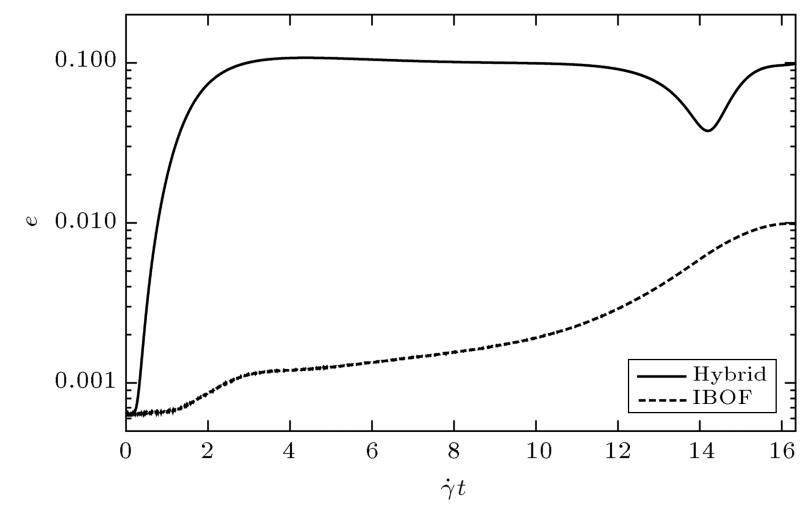

Figure 7. Scalar error, $e$, versus dimensionless time in a half period for non-Brownian particles with $r=0.2$ in a simple shear flow.

The scalar error measure defined in Eq. (39) is used to assess the accuracy of the closure models in reproducing the exact solution.

Figure 7 shows scalar error, $e$, versus dimensionless time, $\dot{\gamma} t$, in a half period. The ordinate is in logarithmic scale. At times close to $\dot{\gamma} t=0$, the error is small as we used the same initial condition in all simulations as the exact solution. As time advances, the error increases. The IBOF model is obviously more accurate than the hybrid model; the IBOF error remains under 0.01 , while the hybrid error goes up to 0.1. Furthermore, the sudden drop in the curve shows the wrong prediction of the time period by the hybrid closure. It is also observable in Figure 5 as the result of hybrid closure crosses the exact result at $\dot{\gamma} t \approx 14.5$ due to the wrong prediction of the time period.

Using the direct Monte-Carlo simulator, the orientation of non-Brownian particles with $r=0.1$ at different time instants in a half period is shown in Figure 8 on the unit sphere. The particles orientation is initially uniformly distributed on the surface of the unit sphere. As the time advances, the particles align with the flow, and then start to redistribute themselves till they reach their initial uniform state. This periodic trend repeats with the time period given by Eq. (40). The non-Brownian particles memorize their initial distribution and their evolution in time as they exactly take the same orientation after one period $T$. The direct Monte-Carlo simulator is attractive in a sense that it yields the instantaneous distribution of individual particles, as presented in Figure 8.

We now consider Brownian particles. Hinch and Leal [33] showed that the orientation distribution of Brownian particles asymptotically tends towards a steady state for large times. Figures 9 and 10 present the scalar error in the computation of the second moment for Brownian particles with $r=0.2$ at $P e=$ 100 and $P e=10$, respectively. Although the IBOF model is less accurate for Brownian particles compared to non-Brownian particles, it is still more accurate 


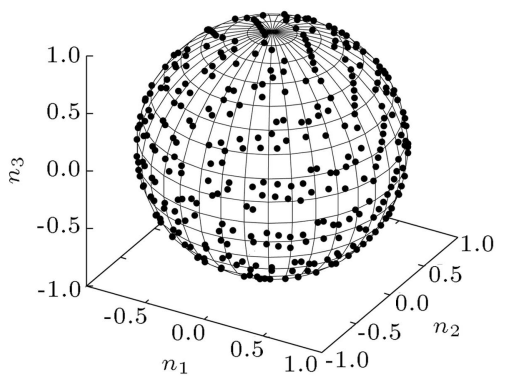

(a) $\dot{\gamma} t=\frac{0}{6} \frac{\dot{\gamma} T}{2}$

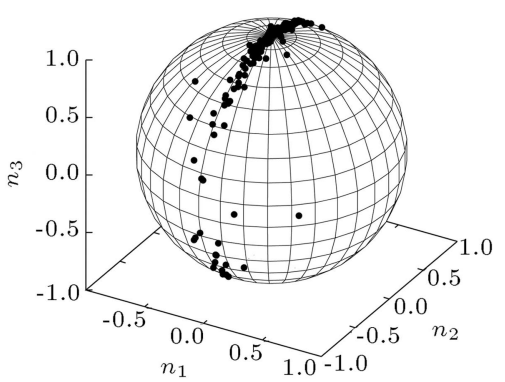

(d) $\dot{\gamma} t=\frac{3}{6} \frac{\dot{\gamma} T}{2}$

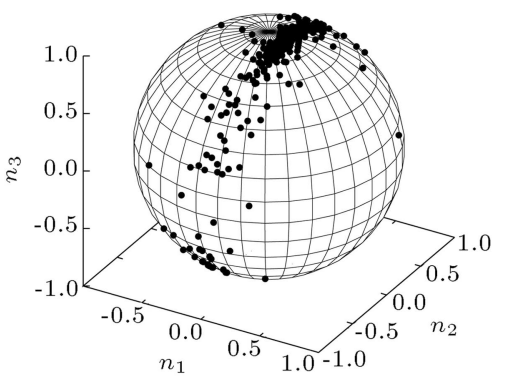

(b) $\dot{\gamma} t=\frac{1}{6} \frac{\dot{\gamma} T}{2}$

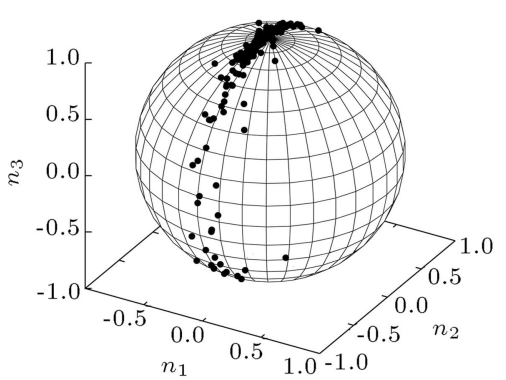

(e) $\dot{\gamma} t=\frac{4}{6} \frac{\dot{\gamma} T}{2}$

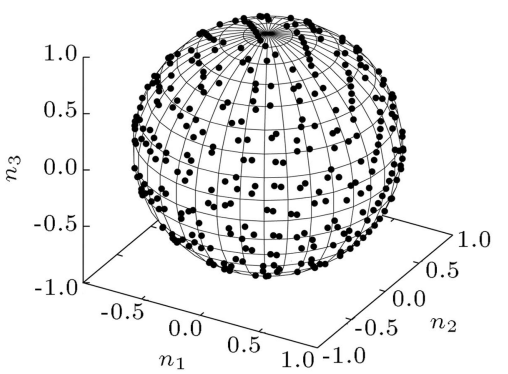

(g) $\dot{\gamma} t=\frac{6}{6} \frac{\dot{\gamma} T}{2}$

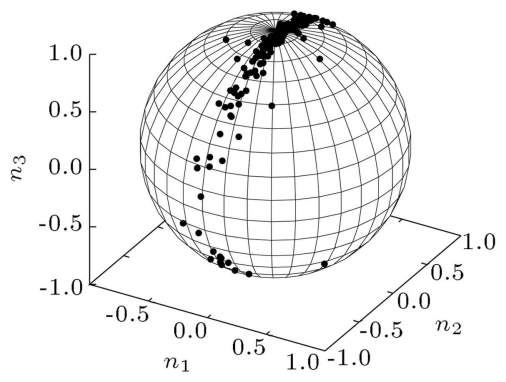

(c) $\dot{\gamma} t=\frac{2}{6} \frac{\dot{\gamma} T}{2}$

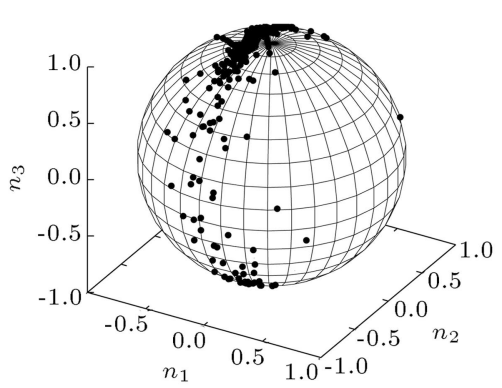

(f) $\dot{\gamma} t=\frac{5}{6} \frac{\dot{\gamma} T}{2}$

Figure 8. Instantaneous particles orientation distribution on the unit sphere from the direct Monte-Carlo simulation with 5000 particles $(r=0.1, P e \rightarrow \infty)$ in dimensionless half period $\dot{\gamma} T / 2$; every 10 th particle is shown.

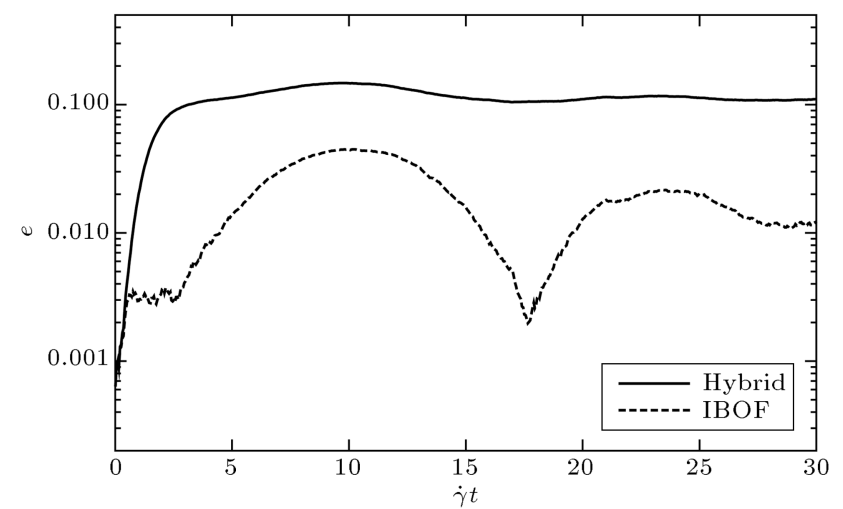

Figure 9. Scalar error, $e$, versus dimensionless time for Brownian particles with $r=0.2$ at $P e=100$ in a simple shear flow.

than the hybrid model. The error tends towards a steady state for Brownian particles. The reason is the wrong prediction of the steady-state value of the second

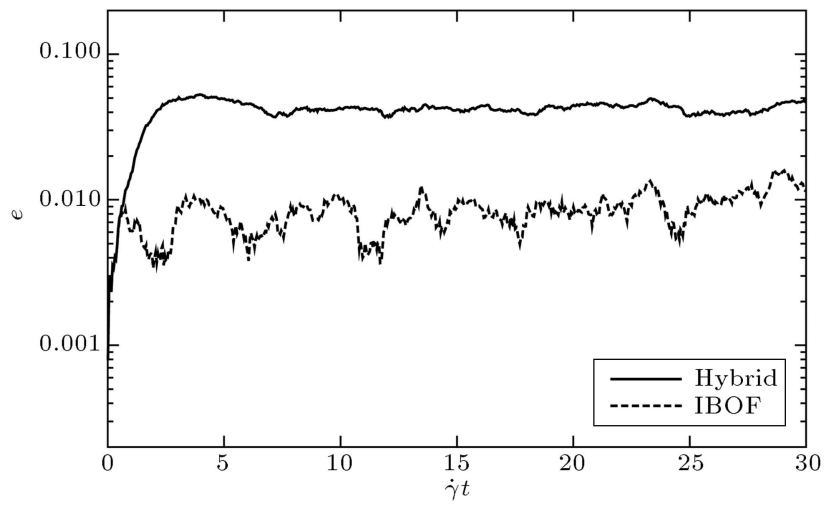

Figure 10. Scalar error, $e$, versus dimensionless time for Brownian particles with $r=0.2$ at $P e=10$ in a simple shear flow.

moment by the closure models. In order to show this fact more obviously, the results of the Monte-Carlo, hybrid and IBOF simulations are shown in Figure 11 


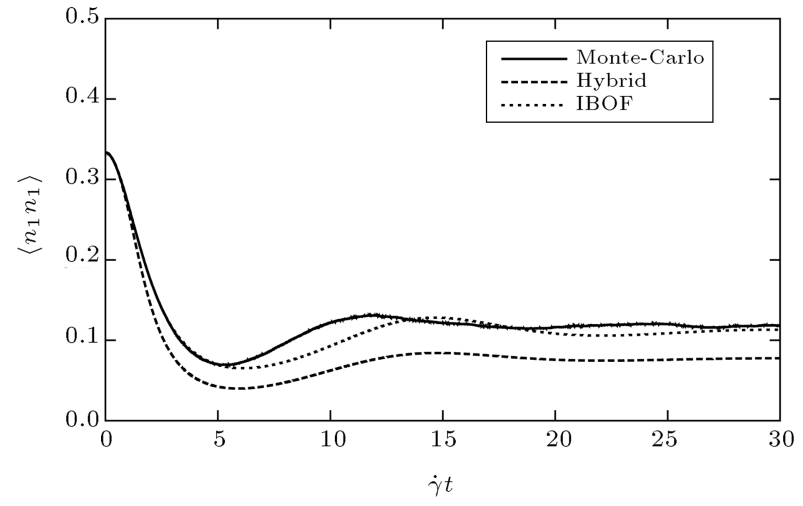

Figure 11. The second moment $\left\langle n_{1} n_{1}\right\rangle$ of orientation distribution function of Brownian particles with $r=0.2$ at $P e=100$.

for the $\left\langle n_{1} n_{1}\right\rangle$ component of the second moment of Brownian particles with $r=0.2$ at $P e=100$.

Frattini and Fuller [31] performed experimental studies of small Brownian rigid rodlike $(r=6.3)$ and disklike $(r=0.45)$ particles at various rotary Péclet numbers suspended in a simple shear flow. By an optical method, they were, for the first time, able to determine the average particle orientation direction projected onto the shear plane and the average degree of alignment $\Delta n^{\prime \prime}$ of the particles. The definition of these two quantities is given in [31]. In order to assess the ability of the computational methods used in the present study to reproduce the experimental data, the degree of alignment $\Delta n^{\prime \prime}$ and the orientation angle for Brownian particles with $r=0.45$ at $P e=$ 144 are shown in Figures 12 and 13 . There is a remarkable similarity between the experimental data and the Monte-Carlo results, especially considering that their experimental material had a rather broad dispersion (about 21\%) in an aspect ratio. Another reason for the discrepancy would be the assumption of inertia-free particles that we made in all of the governing equations for the simulations. However, they

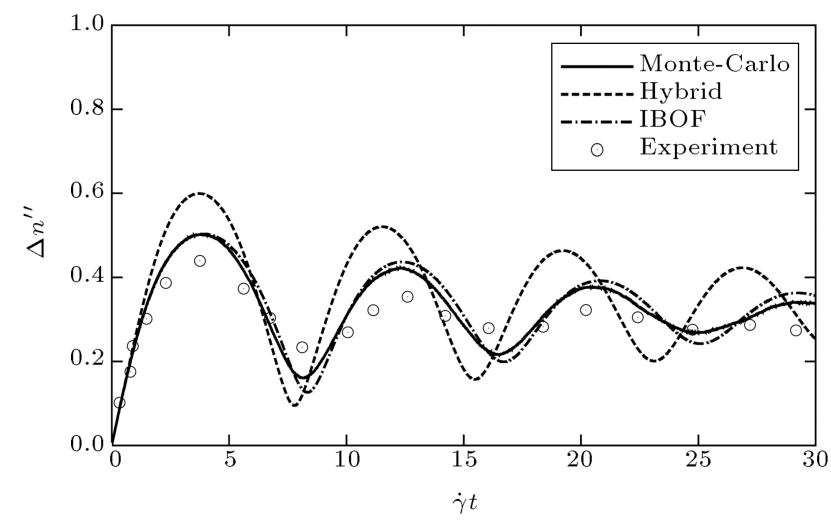

Figure 12. Degree of alignment of Brownian particles with $r=0.45$ at $P e=144$ in a simple shear flow. Experimental data are taken from Figure 12(a) of [31].

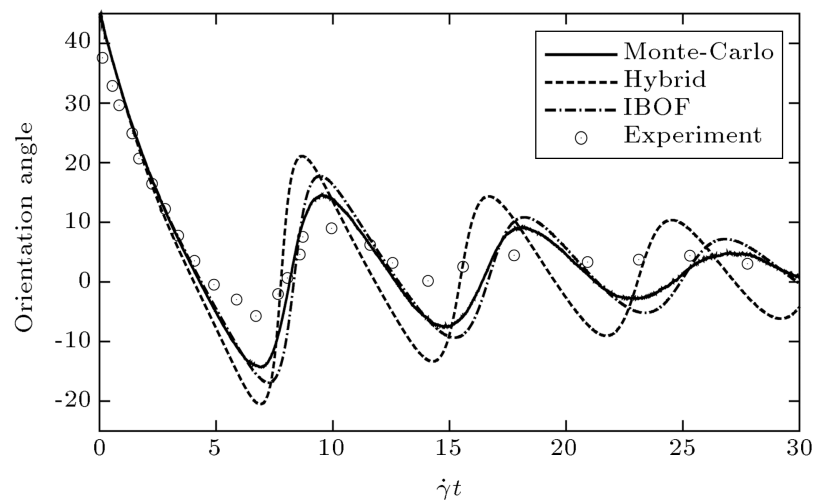

Figure 13. Orientation angle of Brownian particles with $r=0.45$ at $P e=144$ in a simple shear flow. Experimental data are taken from Figure 13(a) of [31].

used the Bentonite particles whose density differs from that of the carrier fluid. The results of the considered closure models stand at a sharp distance from the experiment than those of the Monte-Carlo simulation. The IBOF closure is more accurate.

Figure 14 shows the instantaneous orientation of Brownian particles with $r=0.1$ at $P e=100$ on the unit sphere. Comparison with Figure 8 turns out, as expected, that although the particles are still affected by the external flow, their orientation is distributed more randomly. At $P e=100$, the flow is still strong enough to partially align the particles with the principal shear direction. However, the Brownian motion randomizes the orientation so that the pure periodic behavior of Brownian particles cannot be observed anymore.

The non-Newtonian stress budget of the suspension is also investigated. As explained in Subsection 2.5, the total non-Newtonian stress consists of two parts: the viscous and the elastic stresses. To show the viscoelastic behavior of a dilute suspension of Brownian disklike particles in a simple shear flow, the non-Newtonain, viscous, and elastic stresses are plotted in Figure 15 for $P e \rightarrow \infty, P e=100$, and $P e=10$. As it is expected, the elastic stress vanishes for nonBrownian stress so that the non-Newtonian identically equals its viscous part. As the strength of the Brownian motion increases, the elastic stress becomes more and more significant. Total and partial stress powers are depicted in Figure 16 for $r=0.2$ and $P e=10$. It is observed that the viscous effects are much more pronounced than the elastic effects.

\section{Conclusions}

In this paper, the orientation behavior of a dilute suspension of Brownian rigid disklike particles in a simple shear flow is studied by numerical means. Also, the viscoelasticity of such a suspension is analyzed by considering the stress budget of the two-phase 


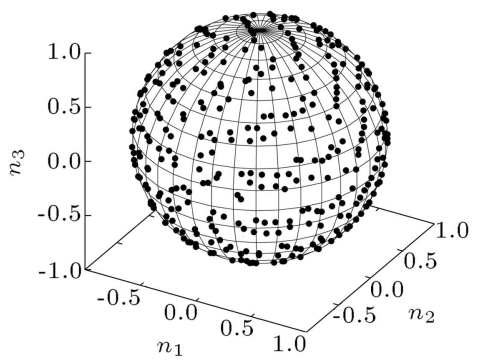

(a) $\dot{\gamma} t=\frac{0}{6} \frac{\dot{\gamma} T}{2}$

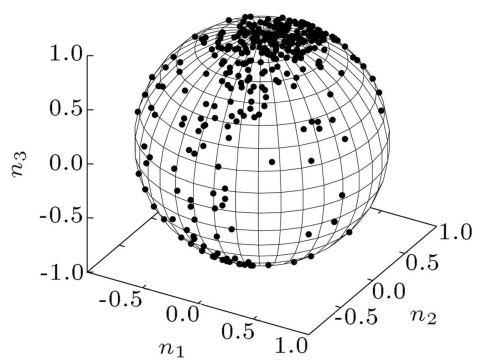

(d) $\dot{\gamma} t=\frac{3}{6} \frac{\dot{\gamma} T}{2}$

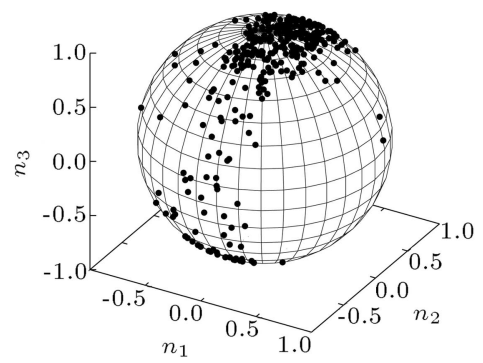

(b) $\dot{\gamma} t=\frac{1}{6} \frac{\dot{\gamma} T}{2}$

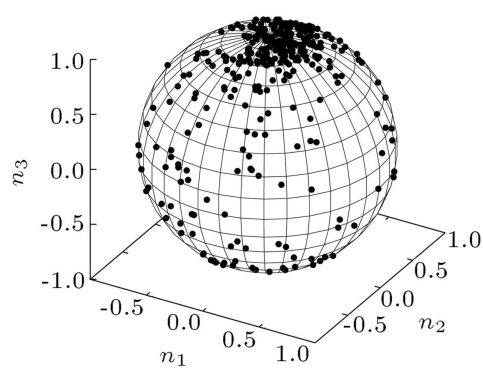

(e) $\dot{\gamma} t=\frac{4}{6} \frac{\dot{\gamma} T}{2}$

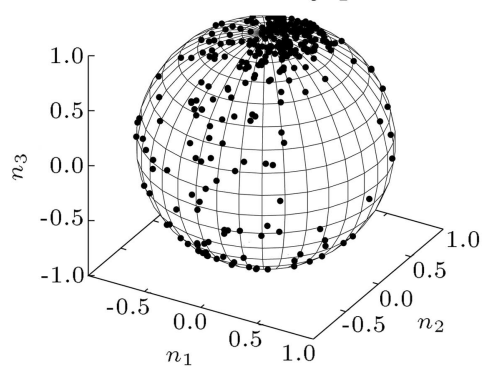

(g) $\dot{\gamma} t=\frac{6}{6} \frac{\dot{\gamma} T}{2}$

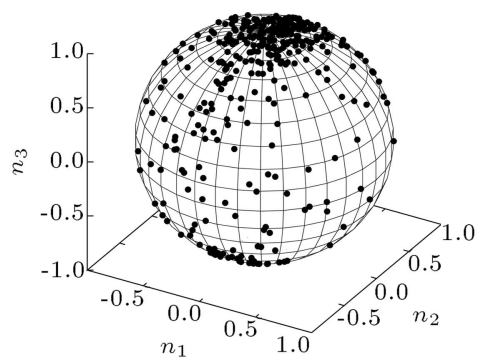

(c) $\dot{\gamma} t=\frac{2}{6} \frac{\dot{\gamma} T}{2}$

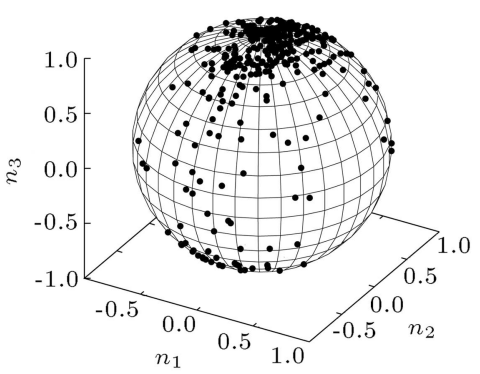

(f) $\dot{\gamma} t=\frac{5}{6} \frac{\dot{\gamma} T}{2}$

Figure 14. Instantaneous orientation distribution of Brownian particles on the unit sphere from the direct Monte-Carlo simulation with 5000 particles $(r=0.1, P e=100)$ in a dimensionless half period $\dot{\gamma} T / 2$ of non-Brownian particles; every 10th particle is shown.

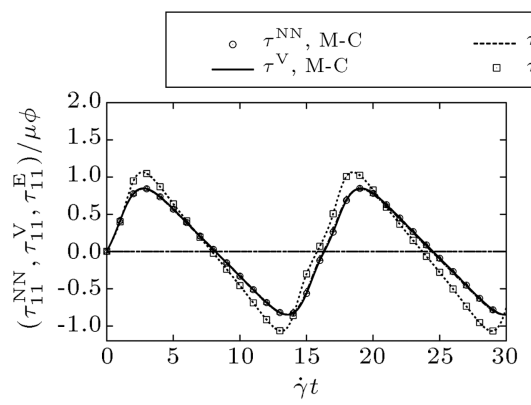

(a) $\mathrm{Pe} \rightarrow \infty$

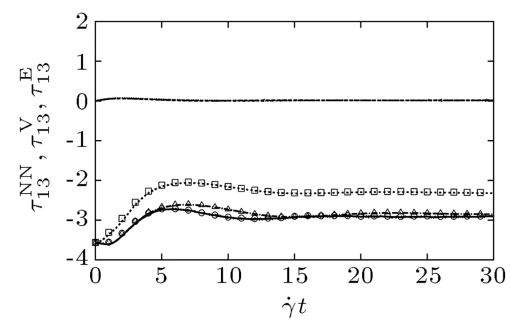

(d) $P e=100$

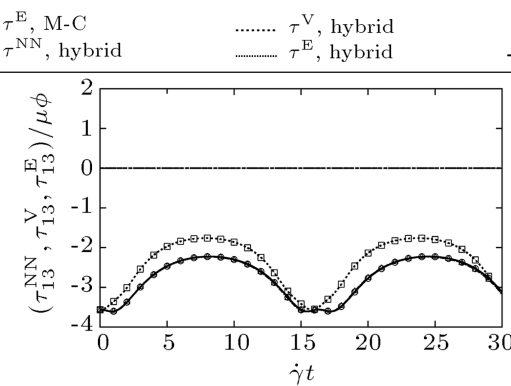

(b) $\mathrm{Pe} \rightarrow \infty$

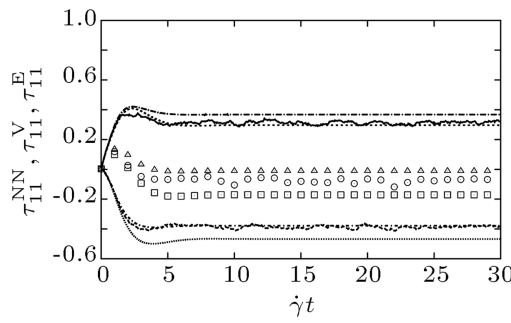

(e) $P e=10$

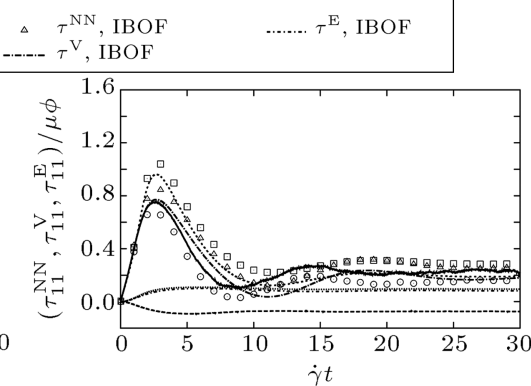

(c) $P e=100$

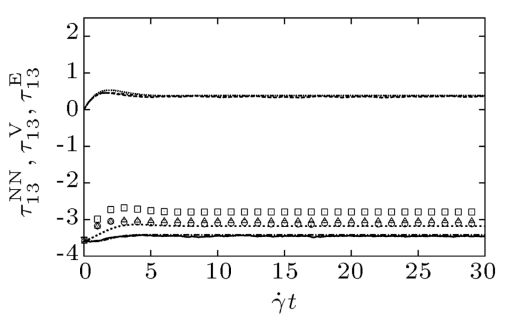

(f) $P e=10$

Figure 15. Non-Newtonian stress budget of Brownian disklike particles with $r=0.2$ in a simple shear flow at various $P e$. 


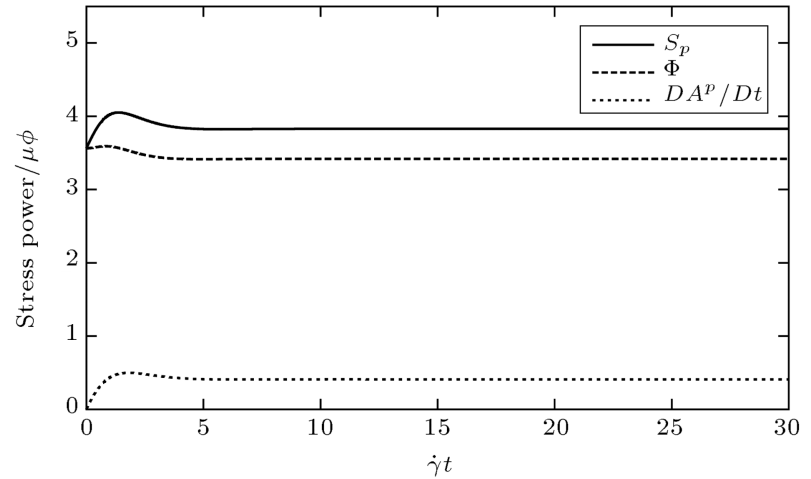

Figure 16. Total, viscous and elastic stress powers of Brownian particles with $r=0.2$ at $P e=10$ in a simple shear flow.

material. Different Péclet numbers and shape factors of particles are considered and various results are presented, e.g. particle orientations in space, viscous and elastic contributions to the non-Newtonian stress tensor, etc. The accuracy of moment approximation method and moment closure models is studied by comparing their results with those of a direct MonteCarlo solver which provides an exact solution. Also, the results are compared with the available experimental and analytical data. It turns out that none of the closure models considered here can exactly reproduce the Monte-Carlo results. The IBOF closure seems to be more accurate than the hybrid closure. Non-Brownian disk, under simple shear flow condition, reveals periodic orientation dynamics in time, whereas the dynamics deviates from periodic behavior by the action of rotary Brownian motion, and aperiodic behavior is observed. Also, the results tend towards a steady-state solution, provided that the rotary Brownian motion is strong enough.

\section{References}

1. Jeffery, G. "The motion of ellipsoidal particles immersed in a viscous fluid", Proc. R. Soc. Lond. A, 102, pp. 161-179 (1922).

2. Brenner, H. "Rheology of a dilute suspension of axisymmetric Brownian particles", Int. J. Multiphase Flow, 1, pp. 195-341 (1974).

3. Okagawa, A., Cox, R. and Mason, S. "The kinetics of flowing dispersions. VI. Transient orientation and rheological phenomena of rods and discs in shear flow", J. Colloid Interface Sci., 45, pp. 303-329 (1973).

4. Risken, H., The Fokker-Planck Equation: Methods of Solution and Applications, Springer-Verlag, Berlin (1989).

5. Öttinger, H., Stochastic Processes in Polymeric Fluids, Springer-Verlag, Berlin (1996).

6. Manhart, M. "Rheology of suspensions of rigid-rod like particles in turbulent channel flow", J. Non-Newtonian Fluid Mech., 112, pp. 269-293 (2003).
7. Manhart, M. "Visco-elastic behaviour of suspensions of rigid-rod like particles in turbulent channel flow", Eur. J. Mech. B/Fluids, 23, pp. 461-474 (2004).

8. Moosaie, A. and Manhart, M. "Direct Monte Carlo simulation of turbulent drag reduction by rigid fibers in a channel flow", Acta Mech., 224, pp. 2385-2413 (2013).

9. Moosaie, A. and Manhart, M. "On the structure of vorticity and near-wall partial enstrophy in fibrous dragreduced turbulent channel flow", J. Non-Newtonian Fluid Mech., 223, pp. 249-256 (2015).

10. Moosaie, A. and Manhart, M. "On the pressure-strain correlation in fibrous drag-reduced turbulent channel flow", Phys. Fluids, 28, p. 025101 (2016).

11. Moosaie, A. and Manhart, M. "A direct numerical simulation method for flow of Brownian fiber suspensions in complex geometries", J. Disper. Sci. Technol., 34, pp. 427-440 (2013).

12. Advani, S.G. and Tucker, C.L. "The use of tensors to describe and predict fiber orientation in short fiber composites", J. Rheol., 31, pp. 751-784 (1987).

13. Hand, G.L. "A theory of anisotropic fluids", J. Fluid Mech., 13, pp. 33-46 (1962).

14. Advani, S.G. and Tucker, C.L. "Closure approximations for three-dimensional structure tensors", $J$. Rheol., 34, pp. 367-386 (1990).

15. Hinch, E. and Leal, L. "Constitutive equations in suspension mechanics. Part 2: Approximate forms for a suspension of rigid particles affected by Brownian rotations", J. Fluid Mech., 76, pp. 187-208 (1976).

16. Chung, D. and Kwon, T. "Invariant-based optimal fitting closure approximation for the numerical prediction of flow-induced fiber orientation," J. Rheol., 46, pp. 169-194 (2002).

17. Paschkewitz, J.S., Dubief, Y., Dimitropoulos, C.D., Shaqfeh, E.S.G. and Moin, P. "Numerical simulation of turbulent drag reduction using rigid fibres", J. Fluid Mech., 518, pp. 281-317 (2004).

18. Paschkewitz, J.S., Dubief, Y. and Shaqfeh, E.S.G. "The dynamic mechanism for turbulent drag reduction using rigid fibers based on Lagrangian conditional statistics", Phys. Fluids, 17, p. 063102 (2005).

19. Moosaie, A., Le Duc, A. and Manhart, M. "Numerical simulation of flow-induced fiber orientation using normalization of second moment", J. Non-Newtonian Fluid Mech., 165, pp. 551-554 (2010).

20. Moosaie, A., Le Duc, A. and Manhart, M. "A priori analysis of a closure model using the reconstruction of the orientation distribution function in flow of fiber suspensions", Comput. Mech., 48, pp. 451-459 (2011).

21. Lipscomb, G.G., Denn, M.M., Hur, D.U., and Boger, D.V. "The flow of fiber suspensions in complex geometries", J. Non-Newtonian Fluid Mech., 26, pp. 297-325 (1988). 
22. Den Toonder, J.M.J., Hulsen, M.A., Kuiken, G.D.C. and Nieuwstadt, F.T.M. "Drag reduction by polymer additives in a turbulent pipe flow: numerical and laboratory experiments", J. Fluid Mech., 337, pp. 193231 (1997).

23. Moosaie, A. and Manhart, M. "An algebraic closure for the DNS of fiber-induced turbulent drag reduction in a channel flow," J. Non-Newtonian Fluid Mech., 166, pp. 1190-1197 (2011).

24. Moosaie, A., Shekouhi, N., Nouri, N.M. and Manhart, M. "An algebraic closure model for the DNS of turbulent drag reduction by Brownian microfiber additives in a channel flow", J. Non-Newtonian Fluid Mech., 226, pp. 60-66 (2015).

25. Moosaie, A. "DNS of turbulent drag reduction in a pressure-driven rod-roughened channel flow by microfiber additives", J. Non-Newtonian Fluid Mech., 232, pp. 1-10 (2016).

26. Yamamoto, T., Suga, T. and Mori, N. "Brownian dynamics simulation of orientational behavior, flowinduced structure, and rheological properties of a suspension of oblate spheroid particles under simple shear", Phys. Rev. E, 72, p. 021509 (2005).

27. Batchelor, G. "The stress system in a suspension of force-free particles", J. Fluid Mech., 41, pp. 545-570 (1970).

28. Hinch, E. and Leal, L. "Constitutive equations in suspension mechanics. Part 1: General formulation", J. Fluid Mech., 71, pp. 481-495 (1975).

29. Fokker, A.D. "Die mittlere Energie rotierender elektrischer Dipole im Strahlungsfeld", Ann. Physik, 43, pp. 810-820 (1914).

30. Planck, M. "An essay on statistical dynamics and its amplification in the quantum theory", Sitzber. Preuß. Akad. Wiss., pp. 324-341 (1917).
31. Frattini, P.L. and Fuller, G.G. "Rheo-optical studies of the effect of weak Brownian rotations in sheared suspensions", J. Fluid Mech., 168, pp. 119-150 (1986).

32. Cintra, J.S. and Tucker, C.L. "Orthotropic closure approximations for flow-induced fiber orientation", $J$. Rheol., 39, pp. 1095-1122 (1995).

33. Hinch, E. and Leal, L. "Time-dependent shear flows of a suspension of particles with weak Brownian rotations", J. Fluid Mech., 57, pp. 753-767 (1973).

\section{Biography}

Amin Moosaie received his BSc degree in Mechanical Engineering-Thermofluids from Iran University of Science and Technology (IUST), Tehran, Iran, in 2006. He earned his MSc degree in Computational Mechanics and his PhD degree in Fluid Dynamics and Aerodynamics from Technische Universität München (TUM), Germany, in 2008 and 2011, respectively. He then started post-doctoral studies at TUM in the same subject, during which he took a sabbatical leave to the Polymer Physics Department at the Eidgenössische Technische Hochschule Zürich (ETH- Zurich), Switzerland, for three months in the Summer of 2011. He joined the Mechanical Engineering Department of Yasouj University, Iran, as a faculty member in 2012, where he is currently an Assistant Professor. He serves as an editor of the Coupled Systems Mechanics journal. He was on the scientific board of several domestic and international conferences. He is the Khwarizmi Award laureate of 2006 and has supervised the best MSc thesis of 2015 as elected by the Iranian Society of Mechanical Engineers (ISME). His primary research interests are computational mechanics, continuum mechanics, turbulence simulation (DNS/LES), drag reduction, and polymer dynamics in fluid flows. 